\title{
Tissue Biotype and Its Relation to the Underlying Bone Morphology
}

Jia-Hui Fu, * Chu-Yuan Yeh, ${ }^{*}$ Hsun-Liang Chan, ${ }^{*}$ Nikolaos Tatarakis, * Daylene J.M. Leong, * and Hom-Lay Wang*

Background: Tissue biotypes have been linked to the outcomes of periodontal and implant therapy. The purpose of this study is to determine the dimensions of the gingiva and underlying alveolar bone in the maxillary anterior region and to establish their association.

Methods: Tissue biotypes of 22 fresh cadaver heads were assessed clinically and radiographically with cone-beam computed tomography (CBCT) scans. Maxillary anterior teeth were atraumatically extracted. The thickness of both soft tissue and bone were measured using a caliper to the nearest $0.1 \mathrm{~mm}$ by two calibrated examiners. Probing depths and gingival recession were measured at two points (mid-labial and mid-palatal). Clinical and CBCT measurements of both soft tissue and bone thickness were subsequently compared and correlated.

Results: No statistically significant differences were observed between the clinical and CBCT measurements of both soft tissue and bone thickness except the palatal soft tissue measurements. The labial gingival thickness was moderately associated with the underlying bone thickness measured with CBCT $(\mathrm{R}=0.429 ; P<0.05)$. Gingival recession was not associated with the thickness of both labial gingiva and bone.

Conclusions: CBCT measurements were an accurate representation of the clinical thickness of both labial gingiva and bone. In addition, the thickness of the labial gingiva had a moderate association with the underlying bone radiographically. J Periodontol 2010;81:569-574.

\section{KEY WORDS}

Bone and bones; cone-beam computed tomography; esthetics; gingiva; gingival recession; implant.

\footnotetext{
* Department of Periodontics and Oral Medicine, School of Dentistry, University of Michigan, Ann Arbor, MI.
}

$\mathrm{T}$ The gingival morphology of the maxillary anterior region plays an important role in determining the final esthetic outcome. Tissue biotypes were associated with the outcomes of periodontal therapy, ${ }^{1}$ root coverage procedures, ${ }^{2,3}$ and, implant esthetics. ${ }^{4,5}$ In 1969, Ochsenbein and Ross ${ }^{6}$ indicated that there were two main types of gingiva morphology, namely the scalloped and thin or flat and thick gingiva. They proposed that the contour of the gingiva closely followed the contour of the underlying alveolar bone. The term "periodontal biotype" was later introduced by Seibert and Lindhe ${ }^{7}$ to categorize the gingiva into "thick-flat" and "thin-scalloped" biotypes. Claffey and Shanley ${ }^{1}$ defined the thin tissue biotype as a gingival thickness of $<1.5 \mathrm{~mm}$, and the thick tissue biotype was referred to as having a tissue thickness $\geq 2 \mathrm{~mm}$ (measurements of 1.6 to $1.9 \mathrm{~mm}$ were not accounted for).

It was suggested that gingival or periodontal diseases were more likely to occur in patients with a thin gingival biotype. ${ }^{1}$ Similarly, in implant restorations, the thick-flat tissue biotype was an important factor for a successful esthetic-treatment outcome. ${ }^{8}$ In root-coverage procedures, a flap thickness of 0.8 to $1.2 \mathrm{~mm}$ was associated with a more predictable prognosis. ${ }^{2,3,9}$ An initial gingival thickness was found to be the most significant factor associated with a complete root-coverage procedure. ${ }^{9}$ In a meta-analysis, Hwang and 
Wang ${ }^{3}$ reported a high association between flap thickness and complete root coverage. In immediate single-tooth-implant restorations, patients with "thin-scalloped" mucosa often had more tissue recession. ${ }^{10}$ On the contrary, patients with "thick-flat" mucosa tended to maintain the implant papillae height. ${ }^{11}$ These observations suggested that tissue biotype might be a significant factor influencing esthetic-treatment outcomes.

Many methods were proposed to measure tissue thickness. These include direct measurements, ${ }^{12}$ probe transparency (TRAN), ${ }^{8}$ ultrasonic devices, ${ }^{13}$ and, most recently, cone-beam computed tomography (CBCT). ${ }^{14}$ In the direct method, the tissue thickness was measured using a periodontal probe. ${ }^{12}$ When the thickness was $\geq 1.5 \mathrm{~mm}$, it was categorized as a thick biotype. When the thickness was $<1.5 \mathrm{~mm}$, it was considered a thin tissue biotype. However, this method of measurement had several inherent limitations, such as the precision of the probe, which is to the nearest $0.5 \mathrm{~mm}$, the angulation of the probe during the transgingival probing, and the distortion of the tissue during probing. In the TRAN technique, the gingival biotype was considered thin when the outline of the periodontal probe showed through the gingival margin from inside the sulcus. ${ }^{8}$ The biotype was considered thick if the probe did not show through the gingival margin. Müller et al. ${ }^{13}$ applied a non-invasive ultrasonic device ${ }^{\dagger}$ to measure gingival thickness. This method had several limitations. Most importantly, it was difficult to determine the correct position and attain reproducible measurements. Recently, CBCT scans were used to visualize and measure the thickness of both hard and soft tissues. ${ }^{14}$ However, to the best of our knowledge, there is a paucity of evidence comparing the accuracy of these techniques used to ascertain tissue thickness.

This study aims to determine the thickness of both soft tissue and underlying alveolar bone in the maxillary anterior region and to establish the association between the tissue biotype and the underlying bone morphology.

\section{MATERIALS AND METHODS}

\section{Samples}

Twenty-two fresh frozen cadaver heads (from 16 white males and six white females; mean age: 67.6 years; age range: 33 to 97 years) were used for this investigation, which was conducted from August 8 to 9,2009 . They were donated to the Anatomy Department of the University of Michigan for educational purposes. The cadaver heads were frozen, and no preservatives were added. They were washed and soaked in an antibiotic solution before they were frozen. Prior to the measurements, the heads were thawed overnight. After the heads were thawed, the gingiva was covered with wet gauze to prevent shrinkage, thereby ensuring that the status of the gingiva and bone was in a fresh state. Measurements were completed in $\sim 12$ hours to prevent tissue shrinkage. The selected study area was the maxillary anterior region, which included teeth \#6 through \#11 (denoted according to the Universal/National System). Teeth with subgingival restorations, metal restorations, intracanal prosthesis (e.g., postcores or pins), probing depths $>4 \mathrm{~mm}$, and fractured buccal plates during extractions of the maxillary teeth were excluded from the study.

Two examiners (JHF and CYY) measured the soft tissue thickness, alveolar bone thickness, probing depths, and gingival recession (GR) at the mid-labial and -palatal sites of the selected teeth. Measurements were taken from the labial gingiva (LG), palatal gingiva (PG), labial bone (LB), and palatal bone (PB). Before the start of the study, a calibration session was performed to evaluate intra- and interexaminer reliability in the clinical measurements. Each examiner (JHF and CYY) performed measurements in three specimens, and this process was repeated twice at 1 -hour intervals. Reliability was then determined using Cohen $\kappa$ statistics.

\section{CBCT Measurements}

CBCT scans of the cadaver heads were taken to obtain radiographic measurements of the thickness of both soft tissue and underlying bone. First, the cadaver head was stabilized using a head locator, and a scout view was obtained to verify the region of interest. Subsequently, a scan of the maxilla was obtained using the $\mathrm{CBCT}^{\ddagger}$ machine in the Radiology Department, University of Michigan, by trained clinicians (JHF and CYY) at $120 \mathrm{KVp}$ and $18.66 \mathrm{~mA}$ for $20 \mathrm{sec}$ onds (voxel size: $0.2 \mathrm{~mm}$; grayscale: 14 bits; focal spot: $0.4 \mathrm{~mm}$; and field of view: $16 \times 22 \mathrm{~cm}$ ). The reconstructed images were generated using a computer software package. $\S$ The thickness of both soft and hard tissue were measured at $2.0 \mathrm{~mm}$ below the alveolar bone crest and perpendicular to the inner cortical plate of the tooth socket using the cross-sectional views taken at the midline of the selected teeth (Figs. 1 and 2). All measurements were performed by one examiner (CYY).

\section{Clinical Measurements}

Assessment of tissue biotype. The tissue biotype was evaluated and categorized by the two examiners (JHF and CYY). The evaluation of the tissue biotype was based on the TRAN of the periodontal probel

\footnotetext{
$\dagger$ SDM, Austenal Medizintechnik, Cologne, Germany.

$\ddagger \mathrm{i}$-CAT Cone-Beam Computed Tomography machine, Imaging Sciences International, Hatfield, PA.

$\S$ OsiriX Imaging Software, Pixmeo, Geneva, Switzerland.

॥ PCP-UNC 15 probe, Hu-Friedy, Chicago, IL.
} 


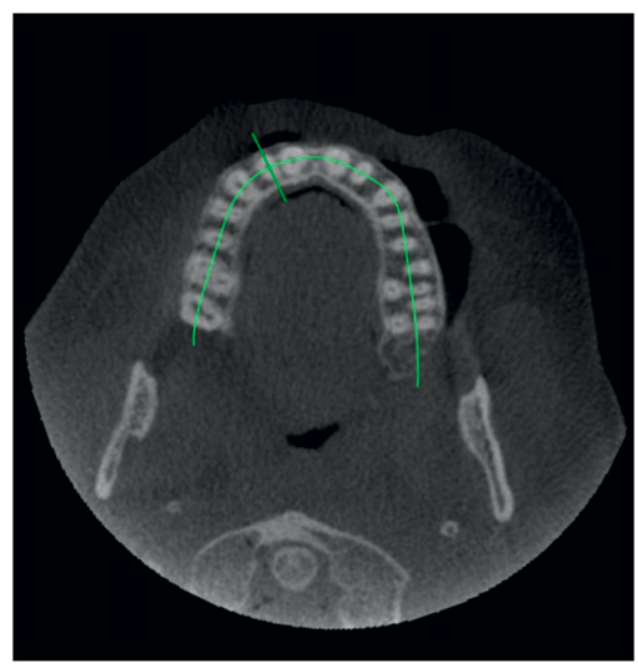

Figure I.

Axial view of a CBCT scan illustrating the area of interest. The vertical green line in the middle of the tooth is the cross-sectional view featured in Figure 2.

through the LG margin. ${ }^{8}$ If the outline of the underlying periodontal probe could be visualized through the gingival margin, it was categorized as a thin biotype; if the outline of the underlying periodontal probe could not be visualized through the gingival margin, it was categorized as a thick biotype.

Probing depth and GR. The probing depth was measured as the distance from the free gingival margin to the base of the pocket. GR was measured as the distance from the cemento-enamel junction to the free gingival margin. All measurements were made to the nearest $0.5 \mathrm{~mm}$ using a periodontal probe."

Thickness of soft tissue and bone. The selected teeth were carefully extracted to ensure that the alveolar bone and soft tissue remained intact. At $2.0 \mathrm{~mm}$ below the alveolar bone crest, the beaks of the caliper ${ }^{\#}$ were positioned perpendicular to the inner wall of the extraction socket on the mid-labial and -palatal surfaces. The measurement obtained was the sum of both soft tissue and bone thickness (SUM). A periosteal elevator ** was used to reflect the soft tissue away from the bone surface. The thickness of the alveolar bone was measured at the same position. The thickness of the soft tissue was obtained from the subtraction of the bone thickness measurement from the SUM measurement.

\section{Statistical Analyses}

All data analyses were performed using a statistical software package. ${ }^{\dagger \dagger}$ Paired $t$ tests were used to compare the data between direct caliper and CBCT methods. Linear regression was used to establish the relationship between both soft tissue and bone thickness. Analysis of variance (ANOVA) and regres-

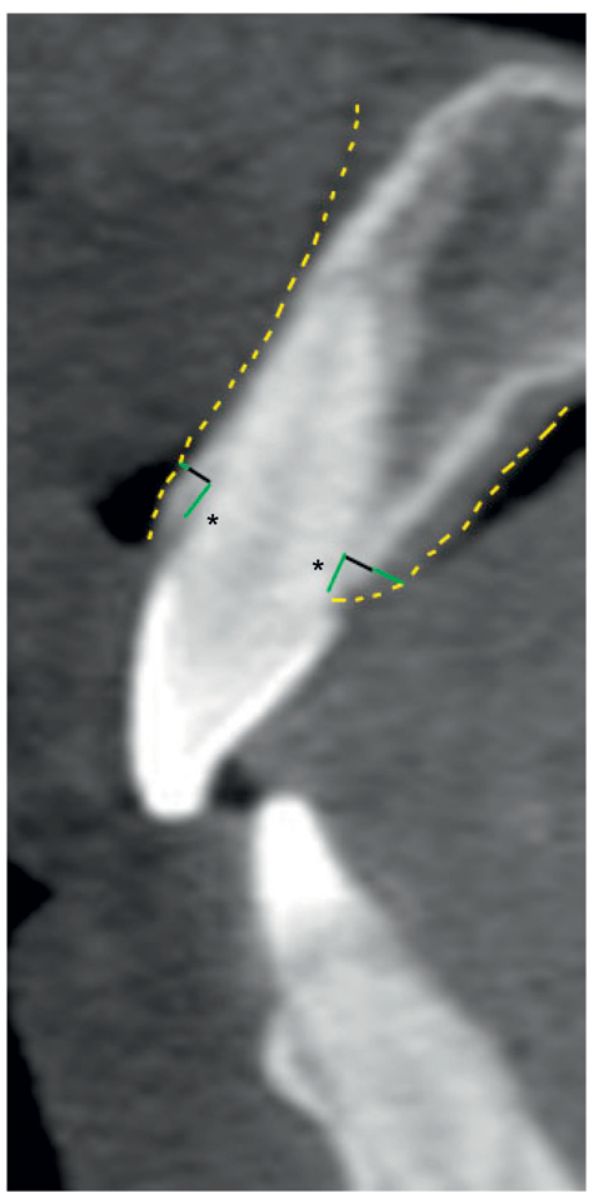

Figure 2.

Cross-sectional view of a CBCT image used to obtain the measurements of both soft tissue and bone thickness of CTLG, CTPG, CTLB, and CTPB. Yellow dashed lines delineate soft tissue margins, vertical green lines illustrate $2.0 \mathrm{~mm}$ from the bone crest (*), horizontal green lines show the soft tissue thickness, and the horizontal black lines represent the bone thickness.

sion analysis were performed to verify the factors associated with tissue biotype or underlying bone morphology. Quantile-quantile plots were charted to determine whether the data collected followed a normal distribution curve. $P<0.05$ was considered statistically significant. The strength of the correlation was determined by the $R$ value: if it was $>0.70$, it was considered a strong correlation; if it was 0.40 to 0.70 , it was a moderate correlation; and if it was $<0.40$, it was a mild correlation. A listwise method was used in the data analysis to compensate for teeth that were excluded.

II PCP-UNC 15 probe, Hu-Friedy.

\# Boley gauge caliper, Salvin Dental Specialties, Charlotte, NC.

** Buser periosteal elevator, Hu-Friedy.

$\dagger \dagger$ SPSS version 16.0, SPSS, Chicago, IL. 


\section{RESULTS}

\section{Intra- and Interexaminer Reliability}

The intraexaminer reliability for both examiners (CYY and JHF) had a $\kappa$ value of 0.8 . The interexaminer reliability for both examiners had a $\kappa$ value of 0.649 (95\% confidence interval: 0.31 to 0.989 ). The intraexaminer reliability (CYY) for the CBCT measurements had a $\kappa$ value of 0.9 . These results demonstrate good intra- and interexaminer reliability for all measurements taken.

\section{Clinical and Radiographic Measurements}

The mean of both soft tissue and bone thickness of the LG, PG, LB, and PB were $0.50 \pm 0.24 \mathrm{~mm}$, $1.00 \pm 0.30 \mathrm{~mm}, 0.83 \pm 0.32 \mathrm{~mm}$, and $1.09 \pm 0.35$ $\mathrm{mm}$, respectively. The corresponding radiographic measurements from the CBCT scans denoted as the CT of the LG, PG, LB, and PB (CTLG, CTPG, CTLB, and CTPB, respectively). The mean of both soft tissue and bone thickness of CTLG, CTPG, CTLB, and CTPB were $0.57 \pm 0.25 \mathrm{~mm}, 1.74 \pm 0.86 \mathrm{~mm}, 0.94 \pm 0.35$ $\mathrm{mm}$, and $1.21 \pm 0.39 \mathrm{~mm}$, respectively (Table 1 ).

\section{Comparison of Measurements Between Caliper and $C B C T$}

There were no statistically significant differences between radiographic and clinical measurements in the groups LG - CTLG, LB - CTLB, and PB - CTPB $(P>0.05)$. However, the PG - CTPG group revealed statistically significant difference, which demonstrates that the palatal soft tissue thickness obtained radiographically and clinically are different (Table 2 ).

\section{Correlation Between Tissue Biotype and Underlying Bone}

A moderate correlation between the CBCT labial thickness of soft tissue and bone (CTLG - CTLB) was established by a simple linear regression model

\section{Table I.}

\section{Labial and Palatal Thickness $(\mathrm{mm})$ of Both Soft Tissue and Alveolar Bone Measured Using a Caliper and CBCT}

\begin{tabular}{cccccc}
\hline Variable & $\mathrm{n}$ & Mean & $\mathrm{SD}$ & Minimum & Maximum \\
\hline Caliper & & & & & \\
LG & 85 & 0.50 & 0.24 & 0.10 & 1.20 \\
PG & 85 & 1.00 & 0.30 & 0.40 & 1.60 \\
LB & 85 & 0.83 & 0.32 & 0.30 & 1.60 \\
PB & 85 & 1.09 & 0.35 & 0.40 & 1.90 \\
CBCT & & & & & \\
CTLG & 100 & 0.57 & 0.25 & 0.20 & 1.86 \\
CTPG & 100 & 1.74 & 0.86 & 0.10 & 4.22 \\
CTLB & 100 & 0.94 & 0.35 & 0.30 & 2.42 \\
CTPB & 100 & 1.21 & 0.39 & 0.50 & 3.30 \\
\hline
\end{tabular}

$(\mathrm{R}=0.429 ; P=0.000)$. Mild correlations existed among the groups LG - LB and CTPG - CTPB $(\mathrm{R}<0.4 ; P<0.05)$ (Table 3).

\section{Association of TRAN and GR With Tissue Biotype}

Statistically significant differences were observed for TRAN on LG and CTLG (Table 4). There was a mild correlation between TRAN and LG and CTLG $(\mathrm{R}<0.4 ; P<0.05)$ (Table 5). No significant relationship was observed between gingival recession and labial soft tissue and bone thickness $(\mathrm{R}<0.4 ; P>0.05)$ (Table 6).

\section{DISCUSSION}

Several methods were attempted to measure the thickness of soft tissue. In a sample population of 31 healthy patients with a mean age of 32 years, Barriviera et al. ${ }^{14}$ showed that the mean canine gingival thickness at $2.0 \mathrm{~mm}$ below the gingival margin was $1.97 \mathrm{~mm}$ using the CBCT method. At $4.0 \mathrm{~mm}$ from the gingival margin in 40 healthy volunteers, Müller et al. ${ }^{15}$ reported that the labial and PG thickness of the maxillary canine gingiva was $0.7 \mathrm{~mm}$ and 2.0 to $4.0 \mathrm{~mm}$, respectively. In the present study, the mean labial soft tissue thickness of the maxillary anterior teeth at $2.0 \mathrm{~mm}$ below the bone crest was $0.5 \mathrm{~mm}$

Table 2.

\section{Comparison of CBCT and Caliper Measurements}

\begin{tabular}{lcc}
\hline Variable & $P$ & Significance \\
\hline LG - CTLG & 0.692 & NSSD \\
PG - CTPG & 0.000 & SSD \\
LB - CTLB & 0.057 & NSSD \\
PB - CTPB & 0.105 & NSSD \\
\hline
\end{tabular}

$\mathrm{SDD}=$ statistically significant difference $(P<0.05) ; \mathrm{NSSD}=$ non-statistically significant difference $(P>0.05)$.

\section{Table 3.}

\section{Association Between Tissue Biotype and Underlying Bone Thickness}

\begin{tabular}{lccccc}
\hline Variable & Coefficient & SE & $t$ & $R$ & $P$ \\
\hline LG - LB & 0.549 & 0.139 & 3.939 & 0.397 & $0.000^{*}$ \\
PG - PB & 0.235 & 0.124 & 1.898 & 0.204 & $0.061^{\dagger}$ \\
CTLG - CTLB & 0.600 & 0.128 & 4.696 & 0.429 & $0.000^{*}$ \\
CTPG - CTPB & 0.135 & 0.044 & 3.080 & 0.297 & $0.003^{*}$ \\
\hline
\end{tabular}

* Statistically significant difference $(P<0.05)$.

$\dagger$ Borderline statistical significance. 
Table 4.

\section{Influence of GR and TRAN on LG, LB, CTLG, and CTLB}

\begin{tabular}{ccccc}
\hline Variable & LG & LB & CTLG & CTLB \\
\hline GR & & & & \\
$F$ & 1.210 & 0.562 & 0.820 & 0.981 \\
$P$ & 0.311 & 0.804 & 0.588 & 0.461 \\
TRAN & & & & \\
F & 7.352 & 3.051 & 9.081 & 1.272 \\
$P$ & $0.009 *$ & 0.086 & $0.004 *$ & 0.264 \\
\hline
\end{tabular}

F stands for the F-test (a ratio of two numbers, where each number estimates a variance).

* Statistically significant difference $(P<0.05)$.

Table 5.

\section{Regression Analysis for LG and CTLG}

\begin{tabular}{lrrrrl}
\hline Variable & Coefficient & SE & \multicolumn{1}{c}{$t$} & $\mathrm{R}$ & \multicolumn{1}{c}{$P$} \\
\hline LG & & & & & \\
GR & -0.046 & 0.038 & -1.214 & 0.064 & 0.229 \\
TRAN & 0.123 & 0.046 & 2.659 & 0.374 & $0.010^{*}$ \\
CTLG & & & & & \\
GR & 0.003 & 0.018 & 0.189 & 0.020 & 0.850 \\
TRAN & 0.141 & 0.048 & 2.916 & 0.287 & $0.004 *$ \\
\hline
\end{tabular}

* Statistically significant difference $(P<0.05)$.

Table 6.

\section{Regression Analysis for GR}

\begin{tabular}{lccccc}
\hline Variable & Coefficient & SE & $t$ & $R$ & $P$ \\
\hline LG & -0.610 & 0.986 & -0.619 & 0.070 & 0.538 \\
LB & -0.757 & 0.617 & -1.225 & 0.137 & 0.224 \\
CTLG & 0.117 & 0.618 & 0.189 & 0.020 & 0.850 \\
CTLB & -0.514 & 0.421 & -1.219 & 0.127 & 0.226 \\
\hline
\end{tabular}

Strong correlation $(R>0.70)$; moderate correlation $(0.70>R>0.40)$; mild correlation $(R<0.40)$.

(range: 0.1 to $1.2 \mathrm{~mm}$ ) clinically and $0.57 \mathrm{~mm}(0.2$ to $1.86 \mathrm{~mm}$ ) radiographically. The mean palatal soft tissue thickness at $2.0 \mathrm{~mm}$ below the bone crest was 1.0 $\mathrm{mm}$ (range: 0.4 to $1.60 \mathrm{~mm}$ ) clinically and $1.74 \mathrm{~mm}$ (range: 0.1 to $4.22 \mathrm{~mm}$ ) radiographically. These results were largely in agreement with the literature. ${ }^{14,15}$

CBCT scans have been widely used for hard tissue imaging because of the superior diagnostic ability obtained through 3-dimensional reconstructive images. Examining the labial-lingual dimension of periodontal defects, Misch et al. ${ }^{16}$ showed that there was no statistically significant difference between the clinical measurements (using a caliper) and radiographic measurements (using CBCT scans). In the present study, CBCT imaging was also used for soft tissue measurements because of the ability to magnify and change the brightness and contrast of the images in the computer program. To better visualize the soft tissue margins, a plastic lip, tongue retractors, and wooden spatulas can be used. ${ }^{14}$

There were no statistically significant differences between radiographic and clinical measurements of both bone and labial soft tissue thickness except in the palatal soft tissue measurements. This could possibly be due to measuring errors such as misangulation of the caliper or overcompression of the soft tissue. ${ }^{17}$ It was difficult to attribute the discrepancy between the direct and CBCT measurements of the PG thickness to either method. The aim of this study is to establish the association between tissue biotype and the underlying bone. It could be preliminarily concluded that CBCT measurements of both bone and labial soft tissue thickness are accurate. CBCT measurements might be a more objective method to determine the thickness of both soft and hard tissue compared to direct measurements.

Because the thickness of the gingival and bone tissues affects the treatment outcomes, possibly due to a difference in the amount of blood supply to the underlying bone ${ }^{18}$ and the susceptibility to resorption, it is important to determine the tissue biotype before the start of the restorative treatment. The linear regression analysis demonstrated a positive moderate correlation between the radiographic thickness of the labial gingiva and its underlying bone (CTLG-CTLB). Clinically, the labial and palatal gingiva were mildly correlated with the underlying bone (LG-LB and PG-PB). Ochsenbein and Ross ${ }^{6}$ believed that long-tapered teeth tend to have a thin-scalloped periodontium, whereas wide-square teeth have thick-flat periodontia. In 1991, Olsson and Lindhe ${ }^{19}$ proposed that long-narrow teeth are more susceptible to GR than short-wide teeth because of the difference in periodontal biotype. In 1993 , Olsson et al. ${ }^{20}$ reported no significant difference between narrow- and widecrown forms with respect to the thickness of the free gingiva. In our study, the thickness of the gingival tissue had a moderate association with the underlying labial alveolar bone, which was a correlation that was initially reported, but not quantified, by Seibert and Lindhe. ${ }^{7}$

There were statistically significant differences noted for TRAN on LG and CTLG. A mild correlation was observed between TRAN and LG, indicating no significant association between visual inspection and periodontal biotype. This supports the conclusion by Eghbali et al. ${ }^{21}$ that TRAN is not a useful method for identifying the gingival biotype. In addition, GR had no 
correlation with LG or CTLG, indicating no significant association between GR and a thin biotype. Hence, GR is less of a predictor of tissue biotype and underlying bone thickness but more of a result of multiple factors such as age, etiology, sample size, and different teeth. ${ }^{22}$ This was consistent with the results described by Müller et al. ${ }^{13}$ in 2000.

\section{CONCLUSION}

The present study demonstrates that the clinical measurements of LG and bone thickness correspond to radiographic measurements, thereby showing that CBCT could be used to determine both soft and hard tissue thickness.

\section{ACKNOWLEDGMENTS}

The authors thank Dean Muller and his staff in the Anatomy Department, University of Michigan, for the organization and transportation of cadaver heads. This study was partially supported by the University of Michigan Periodontal Graduate Student Research Fund. The authors report no conflicts of interest related to this study.

\section{REFERENCES}

1. Claffey N, Shanley D. Relationship of gingival thickness and bleeding to loss of probing attachment in shallow sites following nonsurgical periodontal therapy. J Clin Periodontol 1986;13:654-657.

2. Huang LH, Neiva RE, Wang HL. Factors affecting the outcomes of coronally advanced flap root coverage procedure. J Periodontol 2005;76:1729-1734.

3. Hwang D, Wang HL. Flap thickness as a predictor of root coverage: A systematic review. J Periodontol 2006; 77:1625-1634.

4. Zigdon H, Machtei EE. The dimensions of keratinized mucosa around implants affect clinical and immunological parameters. Clin Oral Implants Res 2008;19: 387-392.

5. De Rouck T, Eghbali R, Collys K, De Bruyn H, Cosyn J. The gingival biotype revisited: Transparency of the periodontal probe through the gingival margin as a method to discriminate thin from thick gingiva. $J$ Clin Periodontol 2009;36:428-433.

6. Ochsenbein C, Ross S. A reevaluation of osseous surgery. Dent Clin North Am 1969;13:87-102.

7. Seibert JL, Lindhe J. Esthetics and periodontal therapy. In: Lindhe J, ed. Textbook of Clinical Periodontology. 2nd ed. Copenhangen, Denmark: Munksgaard; 1989:477-514.

8. Kan JY, Rungcharassaeng K, Umezu K, Kois JC. Dimensions of peri-implant mucosa: An evaluation of maxillary anterior single implants in humans. J Periodontol 2003;74:557-562.
9. Baldi C, Pini-Prato G, Pagliaro U, et al. Coronally advanced flap procedure for root coverage. Is flap thickness a relevant predictor to achieve root coverage? A 19-case series. J Periodontol 1999;70:10771084.

10. Evans CD, Chen ST. Esthetic outcomes of immediate implant placements. Clin Oral Implants Res 2008;19: 73-80.

11. Romeo E, Lops D, Rossi A, Storelli S, Rozza R, Chiapasco M. Surgical and prosthetic management of interproximal region with single-implant restorations: 1-year prospective study. J Periodontol 2008; 79:1048-1055.

12. Greenberg J, Laster L, Listgarten MA. Transgingival probing as a potential estimator of alveolar bone level. J Periodontol 1976;47:514-517.

13. Müller HP, Heinecke A, Schaller N, Eger T. Masticatory mucosa in subjects with different periodontal phenotypes. J Clin Periodontol 2000;27:621-626.

14. Barriviera M, Duarte WR, Januário AL, Faber J, Bezerra AC. A new method to assess and measure palatal masticatory mucosa by cone-beam computerized tomography. J Clin Periodontol 2009;36:564568.

15. Müller HP, Schaller N, Eger T, Heinecke A. Thickness of masticatory mucosa. J Clin Periodontol 2000;27: 431-436.

16. Misch KA, Yi ES, Sarment DP. Accuracy of cone beam computed tomography for periodontal defect measurements. J Periodontol 2006;77:1261-1266.

17. Feldkamp LA, Goldstein SA, Parfitt AM, Jesion G, Kleerekoper $M$. The direct examination of three-dimensional bone architecture in vitro by computed tomography. J Bone Miner Res 1989;4:3-11.

18. Kennedy JE. Effect of inflammation on collateral circulation of the gingiva. J Periodontal Res 1974;9: 147-152.

19. Olsson M, Lindhe J. Periodontal characteristics in individuals with varying form of the upper central incisors. J Clin Periodontol 1991;18:78-82.

20. Olsson M, Lindhe J, Marinello CP. On the relationship between crown form and clinical features of the gingiva in adolescents. J Clin Periodontol 1993;20: 570-577.

21. Eghbali A, De Rouck T, De Bruyn H, Cosyn J. The gingival biotype assessed by experienced and inexperienced clinicians. J Clin Periodontol 2009;36:958963.

22. Smith RG. Gingival recession. Reappraisal of an enigmatic condition and a new index for monitoring. J Clin Periodontol 1997;24:201-205.

Correspondence: Dr. Hom-Lay Wang, Department of Periodontics and Oral Medicine, School of Dentistry, University of Michigan, 1011 N. University Ave., Ann Arbor, MI 48109-1078. Fax: 734/936-0374; e-mail: homlay@umich.edu.

Submitted October 21, 2009; accepted for publication December 1, 2009. 